\title{
Infrared Target Tracking Using Naïve-Bayes-Nearest-Neighbor
}

\author{
Shujuan $\mathrm{GAO}^{\dagger}$, Insuk $\mathrm{KIM}^{\dagger}$, Nonmembers, and Seong Tae JHANG ${ }^{\dagger \mathrm{a})}$, Member
}

\begin{abstract}
SUMMARY Robust yet efficient techniques for detecting and tracking targets in infrared (IR) images are a significant component of automatic target recognition (ATR) systems. In our previous works, we have proposed infrared target detection and tracking systems based on sparse representation method. The proposed infrared target detection and tracking algorithms are based on sparse representation and Bayesian probabilistic techniques, respectively. In this paper, we adopt Naïve Bayes Nearest Neighbor (NBNN) that is an extremely simple, efficient algorithm that requires no training phase. State-of-the-art image classification techniques need a comprehensive learning and training step (e.g., using Boosting, SVM, etc.) In contrast, non-parametric Nearest Neighbor based image classifiers need no training time and they also have other more advantageous properties. Results of tracking in infrared sequences demonstrated that our algorithm is robust to illumination changes, and the tracking algorithm is found to be suitable for real-time tracking of a moving target in infrared sequences and its performance was quite good.

key words: target detection, Naïve Bayes Nearest Neighbor, infrared sequences, night vision
\end{abstract}

\section{Introduction}

Recently, target detection and tracking techniques in infrared sequences have become one of the hottest research topics in military and public surveillance fields. Infrared sensors are not only widely used in military but also civil fields. Information that is not available in visual images can be provided from infrared images even in night and bad weather conditions (e.g., fog, haze, light rain or dust), since the infrared images are obtained by sensing the radiation in the infrared spectrum. Target tracking has also been used in Advanced Driver Assistance Systems (ADAS). Wang et al. [1] proposed diverse AdaBoost SVM tracking algorithms for target tracking in infrared images. Small target tracking is also an important problem in infrared search and tracking systems. Such a system is essential for military applications to warn about incoming targets such as helicopters, warships and aircraft from a distance [2]. An infrared image may include large smooth regions, which lack first-rate details, but there exits a greater scope of data compression. Infrared images also can provide information that is not accessible in visual images. In target tracking of infrared imagery and video many problems still exist. This is due to a number of key factors like lighting changes (shadow vs. sunny day, indoor/night vs. outdoor), cluttered backgrounds

Manuscript received September 17, 2014.

Manuscript publicized November 18, 2014.

${ }^{\dagger}$ The authors are with the Department of Computer, The University of Suwon, Hwaseong-si Gyeonggi-do 445-743, Korea.

a) E-mail: stjhang@ @uwon.ac.kr (Corresponding author)

DOI: 10.1587/transinf.2014EDL8183 (trees, vehicles, animals), artificial appearances (clothing, portable objects), non-rigid kinematics of pedestrians, camera and object motions, depth and scale changes (child vs. adult), and low video resolution and image quality. Some problems are coped with different forms of sensors such as cameras, radar or both of them.

In this paper, to obtain robust and efficient tracking, we analyze the Naïve Bayesian approach for infrared target tracking. The task of NBNN is to determine the most probable class $\widehat{C}$ of a query image $\mathrm{Q}$.

The remainder of this paper is described as follows: Sect. 2 introduces some related works; Sects. 3 and 4 present the proposed target tracking method; Sect. 5 provides the experimental results; and the final section contains the conclusion and ideas for future work.

\section{Related Works}

Considerable research is being conducted by varieties of groups that are designing target detection systems with diverse sensors and computer vision algorithms in order to detect targets or predict the possibility of collisions. There exist various approaches to the problem of target detection and extraction in image sequences. Those methods can be categorized into two major groups: template-based detection and background approaches. Regarding templatebased detection approach, of particular interest in surveillance video processing is detecting humans. In [3], Viola et al. combined images differencing with intensity information to create an AdaBoosted classifier for detecting pedestrians. Popular non-template model not limited to the detection of shapes (e.g., animals), is background subtraction. Foreground regions are identified by contrast of an input image with a background model. The development of efficient and robust background models has attracted a lot of interest from the research community. In the fundamental statistical method, a distribution for each pixel is modeled as a single Gaussian and then any pixels not probably belong to this distribution is considered as a foreground pixel [4]. A background-subtraction method based on nonparametric statistical modeling of pixel process has also been proposed [5]. Elgammal et al. utilized kernel density estimation to acquire pixel intensity distributions.

For tracking approaches, Treptow et al. [6] applied particle filter combined with feature-based and shape-based measurement models to track pedestrians in real time. In 2010, Jiang et al. [7] proposed a method for FLIR image 
segmentation using SVM.

\section{From Image-To-Image to Image-To-Class}

Both methods refer to the classification techniques whose the objective is to find the class that best matches a queried image. Within the Image-To-Image approach, each queried image is compared to all known images and the class of the closest image is selected and assigned to queried image. On the other hand, Image-To-Class approach first pools all of the descriptors of all images belongs to each class to constitute a single representation of that class. The queried image is then compared to all of the classes and the nearest class is selected. Image-To-Class is first used in Naïve Bayes Nearest Neighbor (NBNN) classifier for image classification and has handled dataset with large intra-class variances successfully. Image-To-Image distance is fundamental to Kernel methods (e.g., SVM), notably limits the generalization abilities of non-parametric image classifiers when the number of training images is small. Nearest Neighbor based image classifiers take good production of image classification when the query image is similar to one of the labelled images in classes dataset. Our method is a trivial NN based classifier, which uses Nearest Neighbor distances in the space of the local image descriptors but not in the space of images. NBNN computes straightforward 'Imageto-Class' distances without descriptor quantization.

While images are described as features histograms, the distance between descriptors of images is Image-To-Image distance. The distance can be obtained by KL divergence, which takes the measurements of average log-likelihood of descriptors. Better generalization abilities can be obtained by adopting individual Image-To-Image measurements if we use the descriptor distribution of entire class. By using the KL-distance between the descriptor distributions of $\mathbf{Q}$ and $\mathrm{C}$, such a straightforward Image-to-Class distance can be acquired.

\section{Classification by Naïve Bayes Nearest Neighbor}

The basic idea of our method is described as follows. First, the compressed feature vectors are obtained by compressive sensing method, which we have presented the details of in our previous work [8]. We calculate their distances to the different classes and compare them, as illustrated in Fig. 1. The crucial concept here is to construct the Naïve Bayes assumption of conditional independence of the features given the class number. All descriptors of an image $Q$ are independent and identically distributed given the class of $\mathrm{Q}$. Then the cost to assign $\mathrm{Q}$ to a class $\mathrm{C}$ is patterned as the sum of the lowest costs of assigning each of descriptors $d_{i}$ of $Q$ to C. NN search algorithm finds the closest descriptor of each class $\mathrm{C}$ associating with its distance (cost) to $\mathrm{d}_{\mathrm{i}}$. A class $\hat{\mathrm{C}}$ with the lowest total cost is chosen as the classified class for Q. The algorithm can be summarized as follow:

Each image $\mathrm{Q}$ is classified as is owned by class $\widehat{C}$ according to

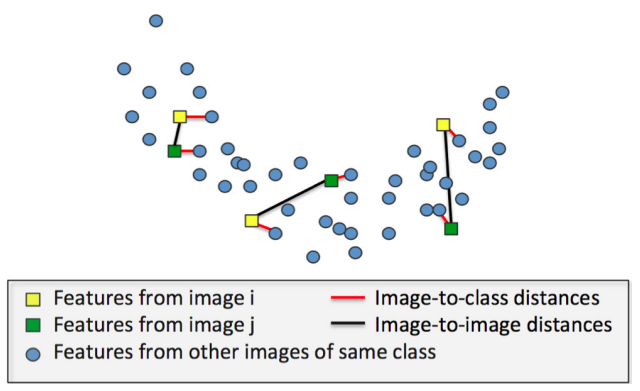

Fig. 1 The key for NBNN: the distances to the class distribution are similar even if the features of the two images are not very the same.

$$
\widehat{C}=\underset{C}{\arg \max } p(C \mid Q)
$$
rule,

Assuming prior class is uniform and applying Bayes

$$
\widehat{C}=\underset{C}{\arg \max } \log (p(Q \mid C))
$$

Let $d_{1} \ldots d_{n}$ indicate all the descriptors of the query image Q. The assumption of independence of the descriptors $\mathrm{d}_{\mathrm{i}}$ (Naive-Bayes assumption) found in image $\mathrm{Q}$ gives:

$$
p(Q \mid C)=p\left(d_{1}, \ldots, d_{n} \mid C\right)=\prod_{i=1}^{n} p\left(d_{i} \mid C\right)
$$

Taking the log probability of the Maximum Likelihood decision rule we get:

$$
\begin{aligned}
\widehat{C} & =\underset{C}{\arg \max } \log (p(C \mid Q)) \\
& =\underset{C}{\arg \max } \frac{1}{n} \sum_{i=1}^{n} \log p\left(d_{i} \mid C\right)
\end{aligned}
$$

Choosing a Gaussian Kernel and substituting single nearest neighbor approximation of $p\left(d_{i} \mid C\right)$ into Eq. (4) as:

$$
\begin{aligned}
\widehat{C} & =\underset{C}{\arg \max } \sum_{d} p(d \mid Q) \log p\left(d_{i} \mid C\right) \\
& =\underset{C}{\arg \min }(K L(p(d \mid Q) \| p(d \mid C)))
\end{aligned}
$$

where KL is the KL-distance between two probability distributions. Under the Naive-Bayes assumption, the class with the minimum distance from the query image will be found.

\section{Implementation Details}

In the experiments, we performed target tracking using images, which are built by our infrared camera and from the Object Tracking and Classification Beyond the Visible Spectrum (OTCBVS) databases. Our algorithms are run on a $\mathrm{PC}$ with $3.10 \mathrm{GHz} \mathrm{CPU}$ and $8 \mathrm{G}$ memory. We provide some experiments to validate the advantage of the proposed method over the classic method of background subtraction in this section. Software platform is Matlab 2012a.

To validate the performance of our target detection system based on infrared images, we have tested five different infrared image sequences that contain different kinds 


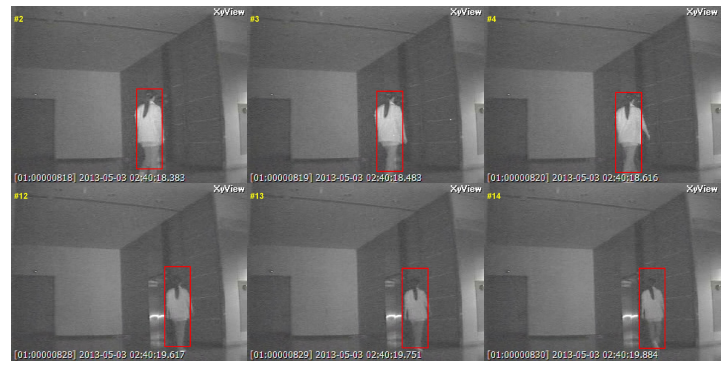

Fig. 2 The tracking results of the first sequence.
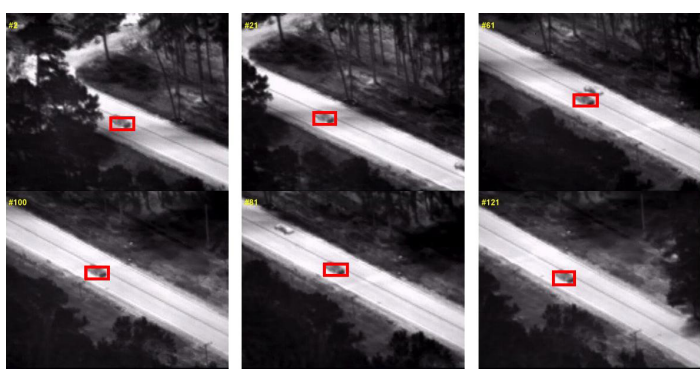

Fig. 3 The tracking results of the second sequence.
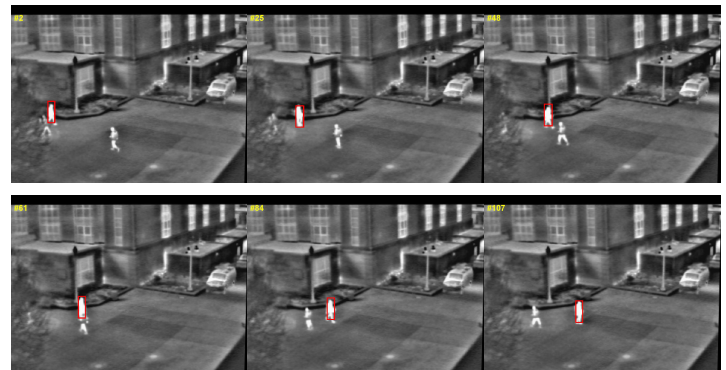

Fig. 4 The tracking results of the third sequence.

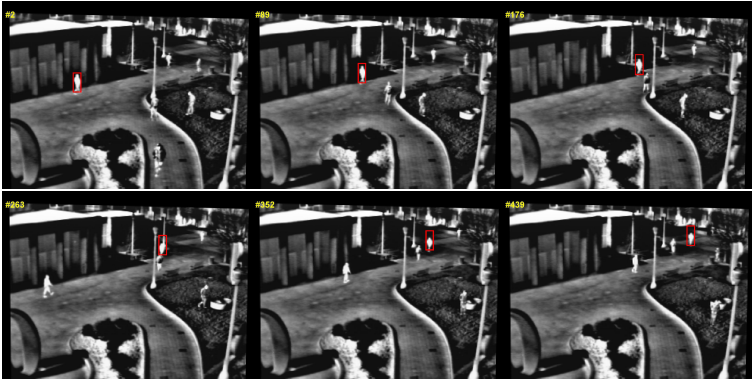

Fig.5 The tracking results of the forth sequence.

of targets. Dataset 1 consists 150 frames $(320 \times 240$ pixels) with a pedestrian that is built up by our infrared camera. Dataset 2, 3, 4 and 5 are provided within the OTCBVS Benchmark Dataset Collection. Dataset 2 consists of 100 frames $(320 \times 256$ pixels $)$ with one car. Dataset 3 consists of 140 frames $(320 \times 240)$. Dataset 4 is for busy pathway intersections on campus consists of 524 frames $(320 \times 240)$ and Dataset 5 is for monitoring an indoor hallway consists of 80 frames $(320 \times 240)$.

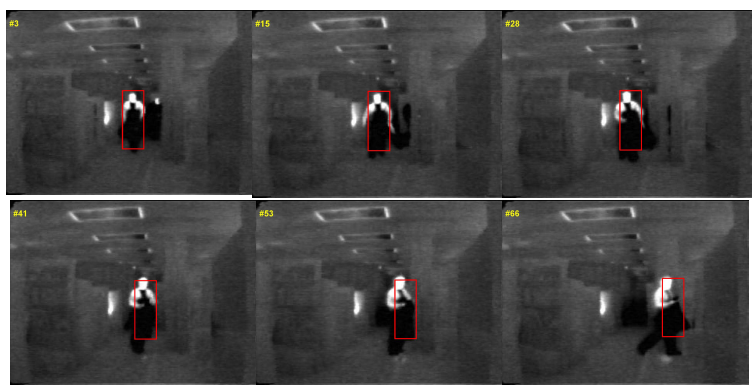

Fig. 6 The tracking results of the fifth sequence.
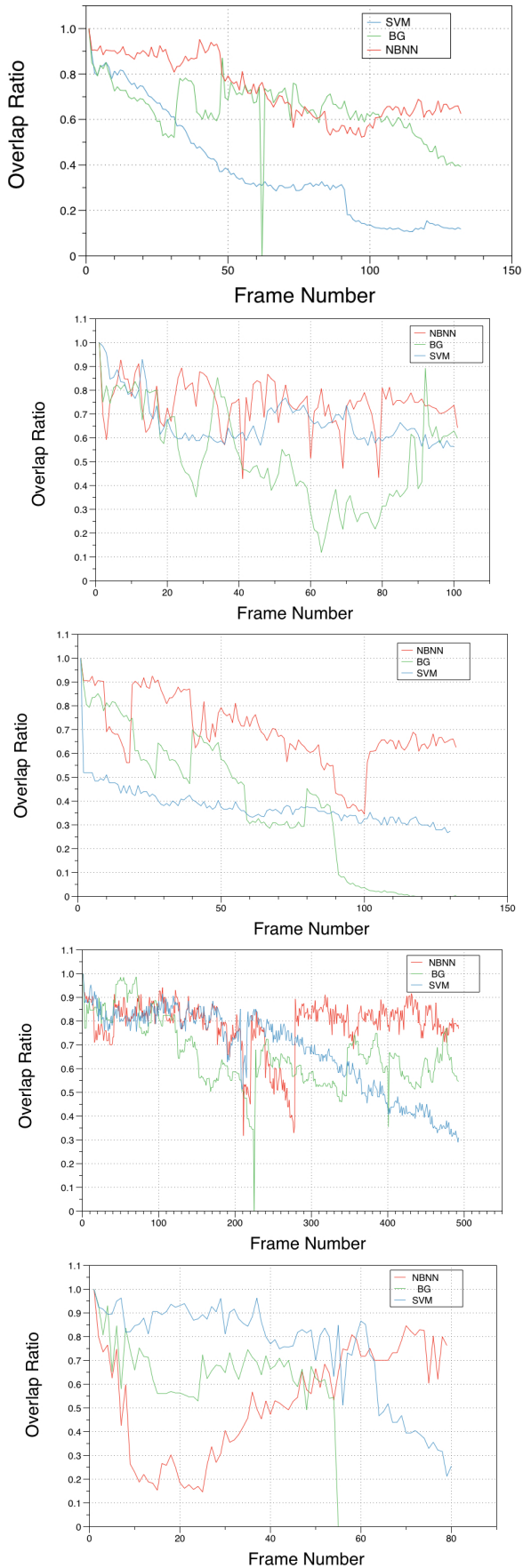

Fig. 7 The comparison of recognition rates (\%) based on Background subtraction (green curves) and SVM (blue curves). 
Many infrared target segmentation algorithms rely on background subtraction techniques [5]. A background subtraction is initially performed to identify local foreground objects. Then gradient information from the objects and the background is combined to generate a contour map representing the confidence for each pixel to belong to a person's boundary [9]. We compared the performance of background subtraction method and SVM which adopted in [7] with our method in infrared images.

Ground truth data for the image sequences are manually labeled for quantitative analysis. The tracking accuracy for each frame is evaluated by overlap ratio, which is defined as the ratio between the area of intersection GT $\cap \mathrm{TR}$ and the area of union GT $\cup \mathrm{TR}$, where GT and TR are the ground truth box and the tracking result box, respectively [10]. Figure 7 shows the overlap ratio of the three algorithms on the sequences. Some of the processed sequences are shown in following figures.

From accuracy curves (Fig. 7) our method (red curves) appears to have more accurate tracking performance than the other two State-of-the-art approaches based tracking system. There was significant drift on frame 62 tested on dataset 1 using the background subtraction method that due to abrupt illumination changes in the background and also may result in unwanted foreground estimates. In sequence $1,3,4$, the background subtraction algorithm cannot track the target correctly and unable to cover the ROIs (actual target). The failure of threshold segmentation due to a big difference between foreground and background, which can conduct to generate a bad target candidate and the actual target drifts away from the reference target when threshold values are similar. Over the course of experiments, SVM represents high algorithm complexity and extensive memory requirements than our method, and the problem of the prediction accuracy deteriorated by noise existing in the images. Our method that made use of the descriptor distribution over an entire class offers better generalization capacity than Image-to-Image.

\section{Conclusion}

This paper introduced a novel method based on Naïve Bayes Nearest Neighbor for object tracking in infrared spectrum.
In this method NBNN is a sparse represented feature based image classifier that achieves an amazing degree of accuracy in Infrared imagery. The experimental results on datasets demonstrate our approach significantly outperforms the classical method based on the background subtraction approach and the SVM. In the future we will pay more attention to improving the accuracy and multi-target identification technology.

\section{Acknowledgments}

This work was supported by GRRC program of Gyeonggi province [(GRRCSUWON2014-B1) Cooperative Recognition and Response System based on Tension Sensing].

\section{References}

[1] Z. Wang, Y. Wu, J. Wang, and H. Lu, "Target tracking in infrared image sequences using diverse AdaBoostSVM,' ICICIC'06, vol.2, pp.233-236, 2006.

[2] T.W. Bae, "Small target detection using bilateral filter and temporal cross product in infrared images," Infrared Physics and Technology, vol.54, no.5, pp.403-411, Sept. 2011.

[3] P. Viola, M.J. Jones, and D. Snow, "Detecting pedestrians using patterns of motion and appearance," Proc. Ninth IEEE International Conference on Computer Vision, 2003, vol.2, pp.734-741, 2003.

[4] C.R. Wren, A. Azarbayejani, T. Darrell, and A.P. Pentland, "Pfinder: Real-time tracking of the human body," IEEE Trans. Pattern Anal. Mach. Intell., vol.19, no.7, pp.780-785, July 1997.

[5] A. Elgammal, D. Harwood, and L. Davis, "Non-parametric model for background subtraction," ECCV, pp.751-767, 2000.

[6] A. Treptow, G. Cielniak, and T. Duckett, "Real-time people tracking for mobile robots using thermal vision," Robotics and Autonomous Systems, vol.54, no.9, pp.729-739, Sept. 2006.

[7] J. Xia, J. Sun, and H. Li, "Forward-looking infrared image segmentation using support vector machine based on feature extraction," 2010 International Conference on Electrical and Control Engineering (ICECE), pp.1031-1034, 2010.

[8] S. Gao, I. Kim, and S.T. Jhang, "Sparse representation based target detection in infrared image," Int. J. Energy, Information and Communications, vol.4, pp.21-28, 2013.

[9] J.W. Davis and V. Sharma, "Robust detection of people in thermal imagery," vol.4, pp.713-716, 2004.

[10] L. HuaPing and S. FuChun, "Fusion tracking in color and infrared images using joint sparse representation," Science ChinaInformation Sciences, vol.55, no.3, pp.590-599, March 2012. 